\title{
Risk Factors Contributing to Microbiological Contamination of Boreholes and Hand Dug Wells Water in the Vina Division, Adamawa, Cameroon
}

\author{
Tangwa Bernard Viban ${ }^{*}{ }^{*}$, Okah-Nnane Ndode Herman ${ }^{1}$, Tangwa Clotilda Layu ${ }^{1}$, \\ Oumarou Palou Madi' ${ }^{1}$, Emmanuel N. Nfor ${ }^{2}$, Manchang Tanyi Kingsly3, Bah Germanus', \\ Ngu Ngwa Victor ${ }^{4}$, Ngakou Albert ${ }^{4}$
}

${ }^{1}$ Institute of Agricultural Research for Development, Ngaoundere, Cameroon

${ }^{2}$ Department of Chemistry, University of Buea, SWR, Buea, Cameroon

${ }^{3}$ Faculty of Agriculture and Veterinary Medicine, University of Buea, Buea, Cameroon

${ }^{4}$ University of Ngaoundere, Ngaoundere, Cameroon

Email: *viban05viban@gmail.com, ndodeherman@gmail.com, tangwaclothildalayu@yahoo.mail.com, paloumadi17@gmail.com, nforemman@gmail.com,manchangtk@justice.com,bahsohg2002@yahoo.com,ngwavictor@yahoo.com, alngakou@yahoo.fr

How to cite this paper: Viban, T.B., Herman, O.-N.N., Layu, T.C., Madi, O.P., Nfor, E.N., Kingsly, M.T., Germanus, B., Victor, N.N. and Albert, N. (2021) Risk Factors Contributing to Microbiological Contamination of Boreholes and Hand Dug Wells Water in the Vina Division, Adamawa, Cameroon. Advances in Microbiology, 11, 90-108.

https://doi.org/10.4236/aim.2021.112007

Received: November 20, 2020

Accepted: February 19, 2021

Published: February 22, 2021

Copyright $\odot 2021$ by author(s) and Scientific Research Publishing Inc. This work is licensed under the Creative Commons Attribution International License (CC BY 4.0).

http://creativecommons.org/licenses/by/4.0/

\begin{abstract}
This study examined the sanitary conditions of public boreholes and hand dug wells water in relation to pathogenic bacteria isolated in water samples. To assess the suitability of drinking water, sanitary inspection of surroundings of the sources was conducted, membrane filtration technic was used to trap the microorganisms in the water sample and other standard microbiological technics were applied to check the heterotrophic plate counts, total coliforms, fecal coliforms and some pathogenic bacteria in the samples. It was observed that there were no delineated protection zones around many boreholes $(61.19 \%)$ and in almost all hand dug wells; thus, hand dug wells were pruned to more contamination than boreholes. Heterotrophic plate counts of boreholes were significantly different between $(\mathrm{p}<0.0001)$ zones in dry and rainy seasons. Total coliforms per $100 \mathrm{ml}$ varied significantly $(\mathrm{p}<0.001)$ between the two seasons both in boreholes and hand dug wells. Fecal coliforms were significantly absent in some boreholes, but significantly present in all hand dug wells with the presence of $E$. coli $157 \mathrm{H}$ in some. Risk assessment of factors contributing to water contamination showed that: pumps manipulation with feet, other sources of contamination such as wastewater dumps, within $10 \mathrm{~m}$ were significantly associated to boreholes contamination with Salmonella spp, Staphylococcus aureuse, fecal coliform ( $\mathrm{p}=0.01)$; in hand dug wells, presence of latrine or septic tank soak-away within $10 \mathrm{~m}$ of the
\end{abstract}


wells, poor depth and lack of internal lining, uncapped wells, presence of trees near the wells, animal and birds feces, were significant and associated to contamination with Salmonela spp., B. cepaceae, S. aureus E. coli $1(\mathrm{p}<0.05)$. Generally water collection points are marked with very poor sanitary conditions and this situation may not change unless the contaminating risk factors are fixed and sanitary measures are taken.

\section{Keywords}

Boreholes Hand Dug Wells, Risk Factors, Sanitation, E. coli, 157H Public Health

\section{Introduction}

There has been encouraging progress with access to safe water and sanitation in both rural and urban areas since the United Nation Water Decade of the 1980s. However, more than 1 billion people around the world still lack access to safe water supply and more than 2.4 billion are without adequate sanitation. In Africa, over one third of the population still remains without access to safe water and sanitation and many of these can only be served by groundwater [1]. The factors leading to contamination are often not well understood, but are frequently ascribed to pollution by on-site sanitation facilities, such as presence pit latrines, as these represent an obvious source of fecal contamination. This is known to be a major cause of disease outbreaks and impact on health [2] [3]. It is further indicated that 1.1 billion people drink water that is at least of "moderate" risk ( $>10$ cfu/ $100 \mathrm{ml}$ Escherichia coli). It is estimated that, by 2030, the African continent may attain $54 \%$ of urban proportion [4]. This phenomenal growth has been qualified as sudden and wild to express the uncontrolled nature of urban growth and the implications it may have on the well-being of city-dwellers. Sub-Saharan Africa is ranked among the world's regions which are mostly at a disadvantage. In Cameroon, contamination of improved water sources is usually a result of poor operation, maintenance leading to frequent failures and lack of environmental education [5]. Consequently, these call concerns about the safety of improved water sources used in communities especially in rural areas where Municipalities are failing to maintain good water quality performance. Diarrhoeal diseases are often caused by contaminated water, poor sanitation, and poor hygiene. In Cameroon, diarrhoeal diseases are the most prevalent waterborne diseases among children aged less than five years. In Yaoundé, for example, the prevalence of diarrhoea is increasing as shown in studies conducted in the city among children aged less than five years, the prevalence increased from $10.8 \%$ in 1998 to $13.1 \%$ in 2004 [6]. In Cameroon and Vina Division in particular, there is lack of monitoring and evaluation strategies of drinking water quality. Hence, poor on-site sanitation and other pollutants are common sources of microbial contamination of groundwater and chemical contaminants. Thus, continuous use of untreated groundwater in the area would pose a public health threat to the 
community [7]. In the Vina Division, sanitation coverage is low; therefore, it is important to identify the sources of groundwater contamination and to assess their individual and combined impact on ground water quality so that interventions to improve water quality are effective.

For these reasons, we assessed the potential sources of contamination of public selected boreholes and hand-dug wells, the suitability of water for drinking from these groundwater sources by analyzing the fecal indicator bacteria and impact on public health.

\section{Materials and Methods}

\subsection{Study Site}

The study was carried in the Vina Division located in the Adamawa Region between latitudes $7^{\circ} 09729$ and $06^{\circ} 53434$ North of the equator and between latitude $014^{\circ} 11.885$ and $013^{\circ} 03.339$ East of the Greenwich Meridian with and, estimated population of more than 1.015.622 inhabitants with almost 17 inhabitants per $\mathrm{km}^{2}$ in 2013 (Zeitlyn, 2018) [8]. Figure 1 indicates the map of Vina Division illustrating localities that were sampled. There are a total of 364 public boreholes based on data obtained from the Divisional delegation, Ministry of Water and Energy. Statistics on the number of public hand dug wells is not known because many are dug by individuals in their homes. Public boreholes are dug by the state and some Non-Governmental Organizations and are unevenly distributed in the divisions that make up the division. Most of these boreholes are poorly managed, hence sanitation controlled around the boreholes is always a problem [9].

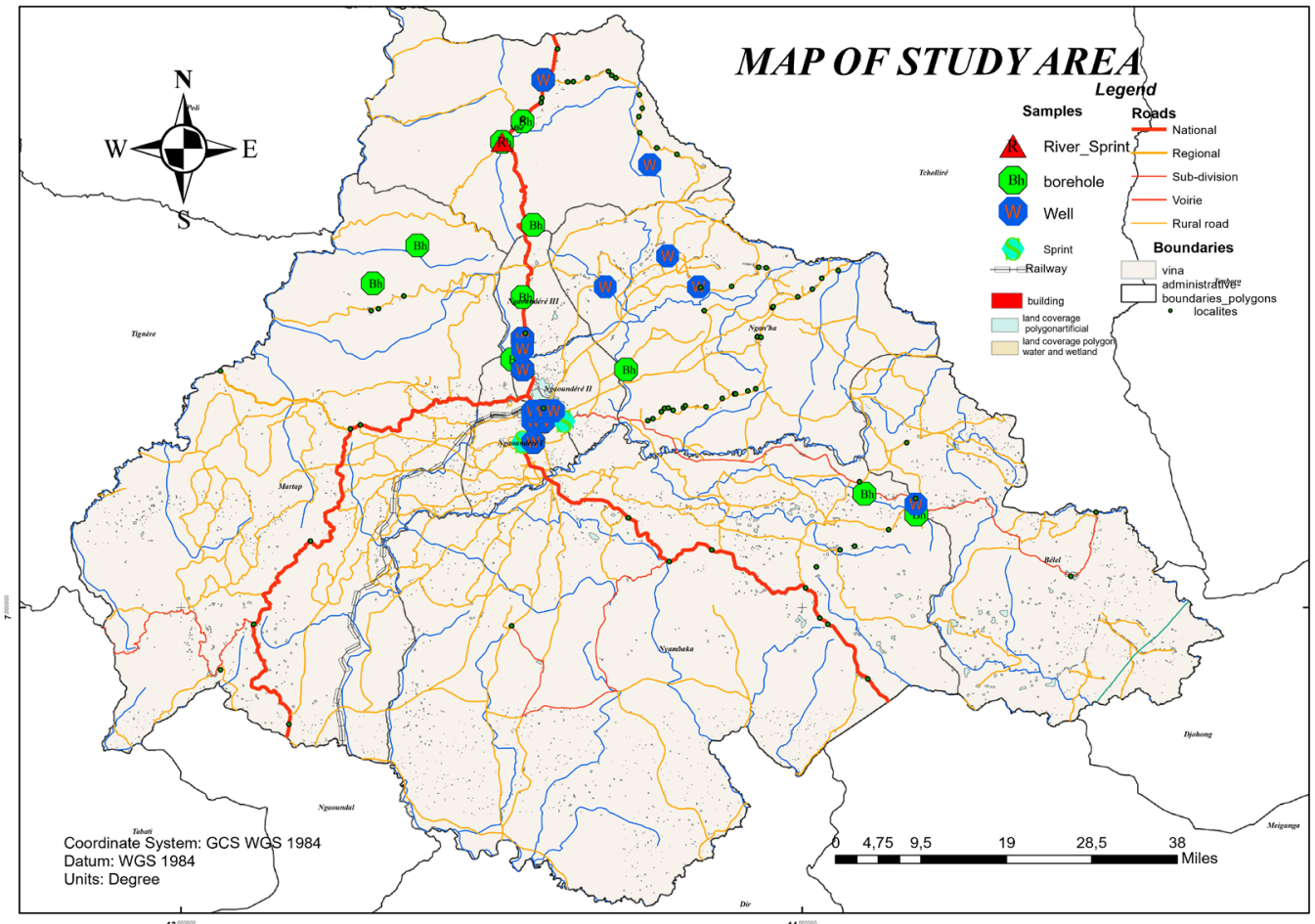

Figure 1. Map of the study area. 


\subsection{Study Design}

A cross sectional survey of water points was carried out during the months of December and April in the dry season and in the months of July and October during the rainy season. Sample points were selected based on the fact that an equivalent water point is the hydraulic unit designed to supply a population of about 300 people with drinking water per day (http://www.iwm-network.org/). Equally distance between the nearest water collection points were taken into consideration. As such 21 public boreholes out of the 364 registered boreholes fulfilled this criterion. Also, 15 hand-dug wells were identified to fulfill this criteria based on a snowball sampling strategy of public wells as most of them were not registered by the Cameroon Ministry of water and energy. The geographic position system of each sampling point was also taken using Garmin etrex 10 GPS.

\subsection{Sanitary Risk Inspection}

A sanitary risk survey involving the identification of potential microbial contamination sources was performed for 21 public boreholes and 15 hand-dug wells in the area to assess their risk level to microbial contamination. The sanitary inspection method was adapted from the British Geological Survey as described by Howard et al. (2003) [10], based on a checklist of 12 factors for boreholes and 10 factors for hand dug wells as indicated in Table 1 and Table 2 respectively. The procedure involved physical inspection of water sources followed by inspection of the surrounding environment and scoring of presumed risk factors (Yes = presence or No $=$ absence) and a few questions asked to people using the water point. A final risk score was obtained by summing the score for each water source.

Table 1. Potential contamination risk factors of boreholes water.

\begin{tabular}{|c|c|c|c|c|}
\hline Receptor & Potential contamination risk factors in dry and rainy seasons & $\begin{array}{l}\text { Boreholes } \\
\text { with risk factor }\end{array}$ & $\begin{array}{l}\text { Contamination/ } \\
\text { risk factor (\%) }\end{array}$ & $\begin{array}{l}\text { Risks by } \\
\text { boreholes (\%) }\end{array}$ \\
\hline \multirow{12}{*}{ 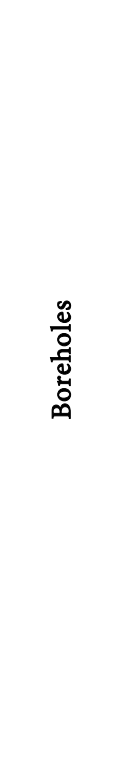 } & Unsanitary/worn-out seal of borehole pumps & 10 & 9 & 47. \\
\hline & Nearest latrine or a pit latrine that percolates to soil & 12 & 10.8 & 57.1 \\
\hline & Trees around boreholes that lead to contamination & 10 & 9 & 47.1 \\
\hline & $\begin{array}{l}\text { Environmental sources of pollution (e.g. rubbish and surface water } \\
\text { discharge) within } 10 \mathrm{~m} \text { radius }\end{array}$ & 8 & 7.2 & 38.09 \\
\hline & No fence around boreholes & 13 & 11.7 & 61.9 \\
\hline & Riverbed near boreholes & 4 & 3.6 & 19.04 \\
\hline & Lack of local management committee & 9 & 8.1 & 42.85 \\
\hline & Pumps manipulated with feet & 4 & 3.6 & 19.04 \\
\hline & Poor drainage near the water point & 9 & 8.1 & 42.86 \\
\hline & Animal and bird excreta & 21 & 18.9 & 100 \\
\hline & $\begin{array}{l}\text { Other nearby sources of contamination such as wastewater dumps trees, } \\
\text { within } 10 \mathrm{~m}\end{array}$ & 3 & 1.8 & 14.28 \\
\hline & & 111 & 99 & \\
\hline
\end{tabular}


Table 2. Potential contamination risk factors of hand dug wells water.

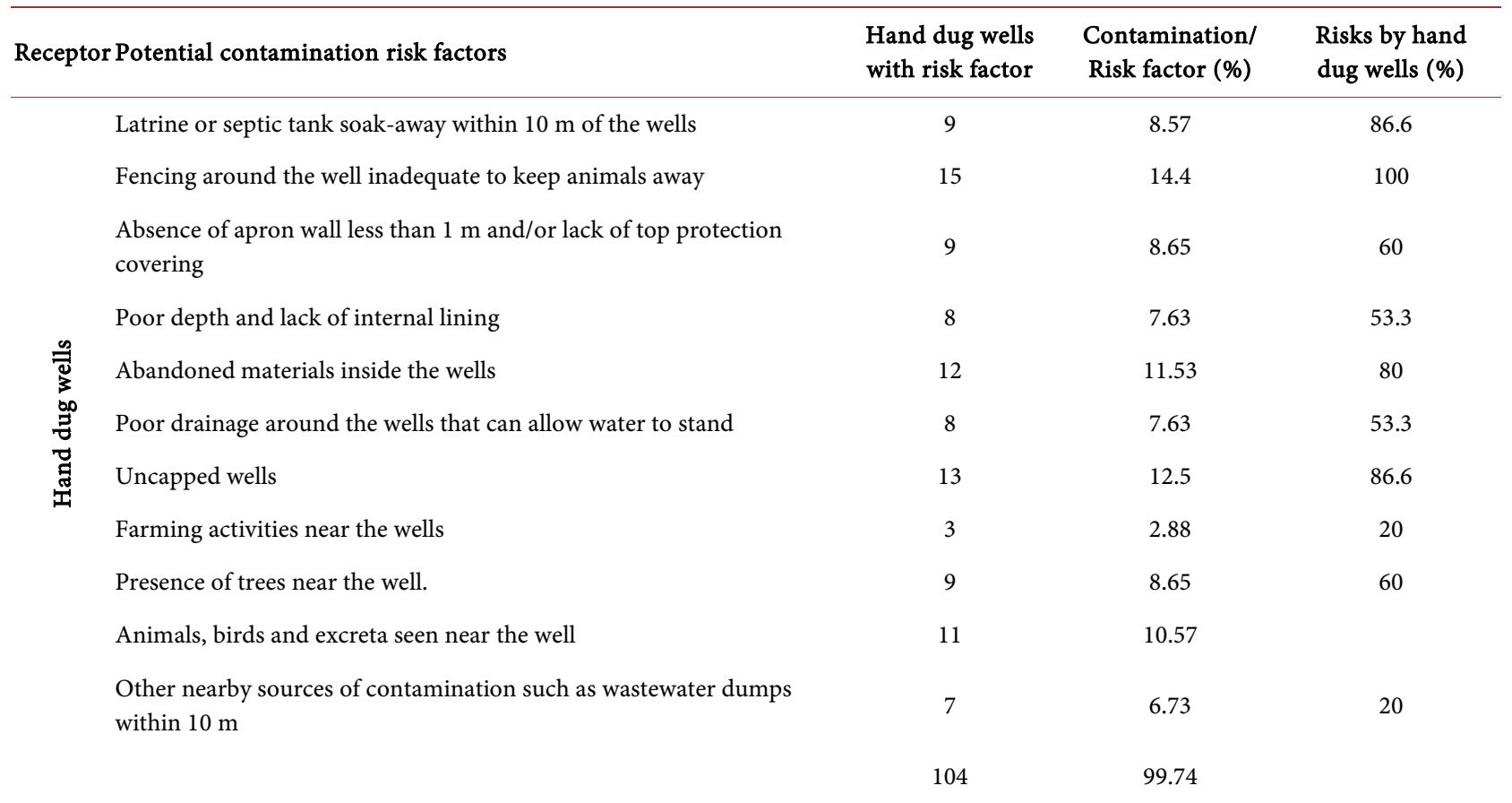

\subsection{Sample Collection and Microbiological Analysis}

Using the membrane filtration technique, each sample was passed through the membrane using a filter funnel and vacuum system. Organisms in samples were then concentrated on the surface of the membrane. Heterotrophic plate count was carried out using a serial dilution and pour plate techniques. M-Endo Les Agar (P.code 101973451, 85766-500G-F) was used for the enumeration of total coliforms, while fecal coliforms were enumerated on m-FC Agar (P.code 101869634, 96961-500G-f). All culture media were prepared following the manufacturer's instructions. E. coli and E. coli 0157.H7 were isolated using Mackonkey sorbitol and confirmed serologically using microgen E. coli 0157 (ref.M44) as rapid confirmatory test for E. coli 0157 .

\subsection{Statistical Analysis}

Water collection points were grouped into zones. The risk of contamination score was calculated as the percentage of total positive answers to the questions posed on the checklist. Average concentrations were calculated using Microsoft excels 2018 and Heterotrophic plate counts expressed as logarithmic transformations $\left(\log _{10}\right)$. Using SPSS, odds ratios were calculated to evaluate relationships between water quality and potential source of contaminations.

\section{Results}

\subsection{Sanitary Inspection Exercise}

Results of the assessment of groundwater sources in the area are summarized for key potential contamination risk factors for 21 boreholes and 15 hand dug wells 
in Table 1 and Table 2 respectively. Seven and eight important risk factors were respectively identified for boreholes and hand dug wells. Results indicated that the quality of water in hand dug wells in the area was more likely to be affected by on-site sanitation facilities than those of the boreholes. The general observation was that there were no delineated protection zones around many boreholes (61.9\%) and almost all hand dug wells. Animal and bird excreta, uncapped wells, Unsanitary/worn-out seal of borehole pumps, no fence around the bore hole and the presence of improper on-site sanitation facilities (silage drains, animal excreta and waste dumps) within $10 \mathrm{~m}$ are the leading key contamination risk factors observed for boreholes and hand dug wells. Site inspection showed that $42.86 \%$ of the boreholes were at risk to poor drainage near the water point. Twelve $(57.1 \%)$ of the boreholes were located close to unlined pit latrines with effluents percolating through the soils, whereas one borehole was within 15 to 20 $m$ of an uncapped well which may itself serve as an entry point of pollutants into the surrounding aquifer. Nine $(42.85 \%)$ of the boreholes lacked local management committees which could control management of the boreholes.

Twelve of the hand dug wells examined (86.6\%) did not have proper top-covering protection, in addition to protection like apron walls. Results also showed that in $60 \%$ of the wells, latrines and septic tank soak ways were sited on the upstream side of the wells and within distances of between 10 to $30 \mathrm{~m}$. All (100\%) of the hand dug wells had no protective fence to block animals and birds from defecating close to the system.

Other important risk factors to contamination observed within the vicinity and in the hand dug wells were complete absence of concrete walls in most of the hand dug wells, or if present were old, with many broken, sticks, old shoes, ropes tied to buckets used for drawing water were normally left at points around the wells that were likely to be source of fecal contamination. Figure 2 illustrates risk contamination of hand dug well and borehole water collection point.

The evaluation of drinking water for presence of bacteria was carried out in both the urban and rural areas of the Vina Division during the dry and rainy seasons. Table 3 and Table 4 indicate respectively the Total Heterotrophic
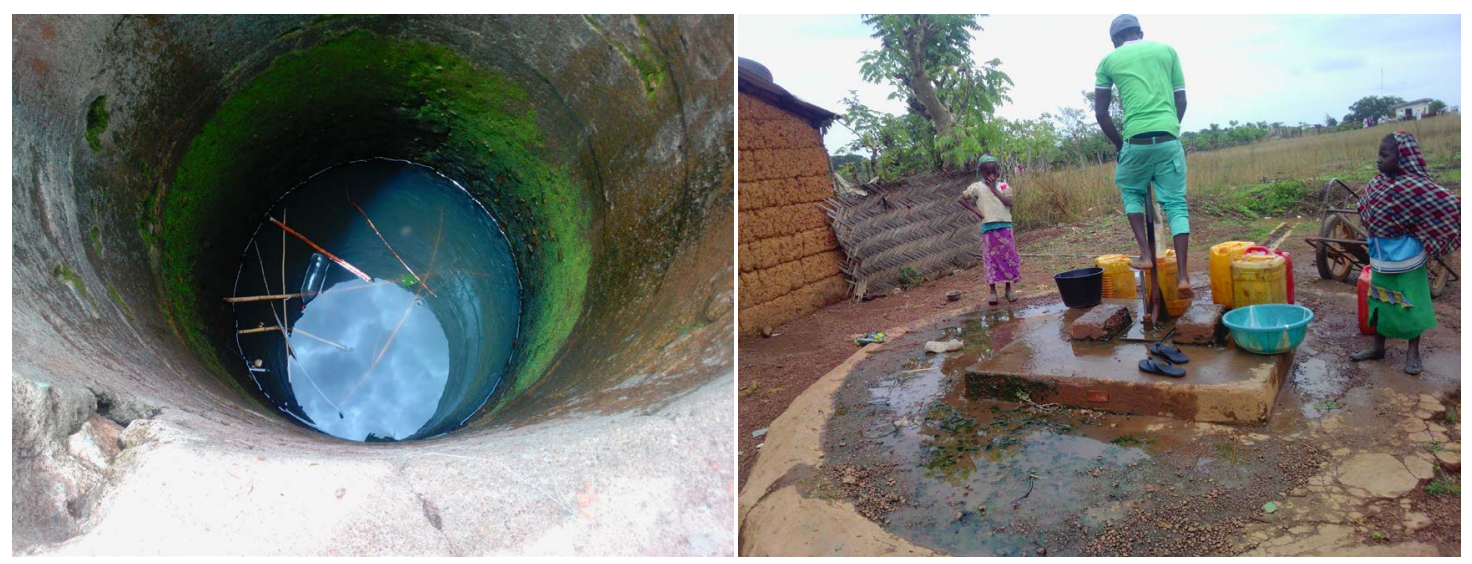

Figure 2. Risk to contamination of hand dug well and borehole water. 
Table 3. Bacteria counts in borehole water from various sampled zones.

\begin{tabular}{|c|c|c|c|c|c|c|c|}
\hline & $\begin{array}{l}\text { Estimated } \\
\text { depths (M) }\end{array}$ & $\begin{array}{c}\text { Fecal coliform count } \\
\text { (dry season) }\end{array}$ & $\begin{array}{l}\text { Serlogicak test for } \\
\text { E. coli } 157 \mathrm{H}\end{array}$ & Risk level & $\begin{array}{l}\text { Fecal coliform count } \\
\text { (rainy season) }\end{array}$ & $\begin{array}{l}\text { Serlogicak test } \\
\text { for } E \text {. coli } 157 \mathrm{H}\end{array}$ & Risk level \\
\hline Zone1 & $36-49$ & $458.3 \pm 227$ & 1colony & & 611 & 1 colony & \\
\hline Zone2 & $35-70$ & $1027.7 \pm 685$ & 0 colony & & 00 & 0 colony & \\
\hline Zone3 & $41-48$ & 00 & 0 colony & & 00 & 0 colony & \\
\hline Zone4 & $36-49$ & $916 \pm 722$ & 0 colony & & 00 & 0 colony & \\
\hline Zone5 & $45-47$ & $13,333 \pm 177$ & 1 colony & & 37 & 0 colony & \\
\hline Zone6 & $42-48$ & 00 & 0 colony & & 00 & 0 colony & \\
\hline Zone7 & $42-47$ & 00 & 0 colony & & 00 & 0 colony & \\
\hline $\mathrm{p}$-value & $<0.1$ & & & & & & \\
\hline
\end{tabular}

Table 4. Bacteria counts in hand dug well water from various sampled zones.

\begin{tabular}{ccccccc}
\hline & $\begin{array}{c}\log _{10} \mathrm{Cfu} / \mathrm{ml} \\
\text { (dry season) }\end{array}$ & $\begin{array}{c}\log _{10} \mathrm{Cfu} / \mathrm{ml} \\
\text { (rainy season) }\end{array}$ & $\begin{array}{c}\text { Total Coliform/100ml } \\
\text { (dry season) }\end{array}$ & $\begin{array}{c}\text { Total Coliform100ml } \\
\text { (rainy season) }\end{array}$ & $\begin{array}{c}\text { Fecal coliform 100ml } \\
\text { (dry season) }\end{array}$ & $\begin{array}{c}\text { Fecal coliform/100ml } \\
\text { (rainy season) }\end{array}$ \\
\hline Zone 1 & $47 \pm 1.94^{\mathrm{a}}$ & $61.62 \pm 0.27^{\mathrm{c}}$ & $8433.33 \pm 1879.01^{\mathrm{a}}$ & $8383.33 \pm 1806.01^{\mathrm{a}}$ & $1833.33 \pm 2160.3^{\mathrm{a}}$ & $1166.67 \pm 888.5 \mathrm{~b}$ \\
Zone 2 & $59.72 \pm 0.32^{\mathrm{d}}$ & $61.43 \pm 0.56^{\mathrm{c}}$ & $10,294.3 \pm 4908.83^{\mathrm{ab}}$ & $3408.33 \pm 1438.2^{\mathrm{a}}$ & $583.33 \pm 280^{\mathrm{a}}$ & $833.33 \pm 455.3 \mathrm{~b}^{\mathrm{a}}$ \\
Zone 3 & $58.55 \pm 1.06^{\mathrm{cd}}$ & $59.16 \pm 0.07^{\mathrm{a}}$ & $19,424.5 \pm 8610.4^{\mathrm{b}}$ & $18,927.0 \pm 5883^{\mathrm{b}}$ & $1083.33 \pm 491.6^{\mathrm{a}}$ & $666.66 \pm 334.5^{\mathrm{a}}$ \\
Zone 4 & $55.58 \pm 0.80^{\mathrm{b}}$ & $60.61 \pm 0.46^{\mathrm{b}}$ & $14,962.3 \pm 6448.4^{\mathrm{ab}}$ & $9139.0 \pm 5883.39^{\mathrm{a}}$ & $3916.67 \pm 1068.5^{\mathrm{b}}$ & $1916.67 \pm 1158.7 \mathrm{c}$ \\
Zone 5 & $57.51 \pm 0.83^{\mathrm{C}}$ & $59.19 \pm 0.15^{\mathrm{a}}$ & $10,040.2 \pm 931.1^{\mathrm{ab}}$ & $9173.5 \pm 221.61^{\mathrm{a}}$ & $2208.33 \pm 2379.2^{\mathrm{ab}}$ & $3333.33 \pm 1158.8^{\mathrm{d}}$ \\
$\mathrm{P}$ & $\mathrm{p}<0.00001$ & $\mathrm{p}<0.00001$ & 0.2503 & $\mathrm{p}<0.001$ & $\mathrm{p}<0.01$ & $\mathrm{p}<0.001$ \\
$\mathrm{~F}$ & $2912.56^{* * *}$ & $67.77^{* * *}$ & 1.44 & $5.41^{* *}$ & 3.99 & $4.65^{* * *}$ \\
T test & $\mathrm{P}=0.07$ & & $\mathrm{P}=0.4$ & & $\mathrm{P}=0.7$ &
\end{tabular}

Note: the averages of different zones that have same subscript letters are not statistically significantly different from each other while the others that have different leters are statistically significantly different at $95 \%$ confident level. ${ }^{* * *}$ Very very significant difference $(\mathrm{p}<0.0001)$; ${ }^{* *}$ very significant difference $(\mathrm{p}$ $<0.001)$, ${ }^{*}$ significant difference $(\mathrm{p}<0.005)$.

Bacteria (THB), Total coliform counts and fecal coliform counts from boreholes water and hand dug wells in the dry and rainy seasons. A very significant $(\mathrm{p}<$ 0.0001) difference between zones in heterotrophic plate count of bacteria load for boreholes water was observed during the dry season. These values ranged from $\log _{10} 42.27 \mathrm{CFU} / \mathrm{ml}$ (zone 1) to $\log _{10} 46.51 \mathrm{CFU} / \mathrm{ml}$ (zone 7), and from $\log _{10}$ $45.14 \pm 1.8 \mathrm{CFU} / \mathrm{ml}$ (zone 1) to $\log _{10} 51.18 \pm 0.12 \mathrm{ml}$ (zone 7) during the rainy season. As for hand-dug wells, THB differed very significantly $(\mathrm{p}<0.00001)$ between zones, varying from $\log _{10} 47 / \mathrm{CFU} / \mathrm{ml}$ (zone 1) to $\log _{10} 59.72 \mathrm{CFU} /$ (zone 2) in the dry season, while in the rainy season, the highest counts recorded was 61.62 CFU/ml (zone 1), while the lowest was $59.16 \mathrm{CFU} / \mathrm{ml}$ (Zone 3). In boreholes water, there was a significant $(\mathrm{p}<0.01)$ difference between the readings of the HTB in the dry season and those of the rainy season. In the hand dug wells, no significance $(\mathrm{p}=0.07)$ difference was noticed in the values obtained between the readings of rainy and dry season.

According to the analysis, the THB was extremely high in zone 3 (46.65 
$\mathrm{cfu} / \mathrm{mL}$ ) for boreholes and $59.72 \mathrm{cfu} / \mathrm{mL}$ for hand dug wells during the dry season. In the rainy season the THB values were higher than those obtained in the dry season. The comparison of heterotrophic plate count of boreholes between the dry and rainy seasons through $\mathrm{T}$-Test revealed statistically significant $(\mathrm{P}<$ 0.010) difference between the seasons.

\subsection{Fecal Coliform Correlation of Hand Dug Wells and Boreholes Water}

In the dry season, boreholes in zones 1,2, 4 and 5 had fecal coliform concentration per $100 \mathrm{ml}$ of more than $100 \mathrm{CFU} / 100 \mathrm{ml}$. while in the rainy season only zone one had fecal coliform counts out of the range as indicated Table 5. In the hand dug wells both in the dry and rainy season, fecal coliform counts varied very significantly $(\mathrm{p}<0.001 ; \mathrm{p}<0.01)$, hence all the hand dug wells water were classified at high risk or very high risk to public health (Table 6). Risk classification levels were done based guideline values for bacteriological water quality water control as seen in Table 7.

From this result we can conclude that there is systematic pollution of groundwater in the study area. However, precise recognition of Enteropathogenic E. coli (EPEC), classically associated with outbreaks of infant diarrhea [11] remains

Table 5. Risk level classification of boreholes based on contamination with fecal coliforms.

\begin{tabular}{|c|c|c|c|c|c|c|c|}
\hline & $\begin{array}{l}\text { Estimated } \\
\text { depths }(\mathrm{M})\end{array}$ & $\begin{array}{c}\text { Fecal coliform count } \\
\text { (dry season) }\end{array}$ & $\begin{array}{l}\text { Serlogical test for } E \text {. } \\
\text { coli } 157 \mathrm{H}\end{array}$ & Risk level & $\begin{array}{c}\text { Fecal coliform count } \\
\text { (rainy season) }\end{array}$ & $\begin{array}{l}\text { Serlogical test } \\
\text { for E. coli } 157 \mathrm{H}\end{array}$ & Risk leve \\
\hline Zone 1 & $36-49$ & $458.3 \pm 227.2$ & 1colony & & 611 & 1 colony & \\
\hline Zone 2 & $35-70$ & $1027.7 \pm 685.4$ & 0 colony & & 00 & 0 colony & \\
\hline Zone 3 & $41-48$ & 00 & 0 colony & & 00 & 0 colony & \\
\hline Zone 4 & $36-49$ & $916 \pm 722$ & 0 colony & & 00 & 0 colony & \\
\hline Zone 5 & $45-47$ & $13,333 \pm 177.5$ & 1 colony & & 37 & 0 colony & \\
\hline Zone 6 & $42-48$ & 00 & 0 colony & & 00 & 0 colony & \\
\hline Zone 7 & $42-47$ & 00 & 0 colony & & 00 & 0 colony & \\
\hline $\mathrm{p}$-value & $<0.1$ & & & & & & \\
\hline
\end{tabular}

Table 6. Risk level classification of hand dug wells based on contamination with fecal coliforms.

\begin{tabular}{|c|c|c|c|c|c|c|c|}
\hline & $\begin{array}{l}\text { Estimated } \\
\text { depth }(\mathrm{M})\end{array}$ & $\begin{array}{c}\text { Fecal coliform count } \\
\text { (dry season) }\end{array}$ & Risk level & $\begin{array}{l}\text { Serlogical test } \\
\text { for E. coli } 157 \mathrm{H}\end{array}$ & $\begin{array}{c}\text { Fecal coliform count } \\
\text { (rainy season) }\end{array}$ & $\begin{array}{l}\text { Serlogical test for } \\
\text { E. coli } 157 \mathrm{H}\end{array}$ & Risk level \\
\hline Zone 1 & $4-25$ & $1833.33 \pm 216 ., 3^{\mathrm{a}}$ & & 2 colonies & 1166 & 2 colonies & \\
\hline Zone 2 & $10-25$ & $583.33 \pm 280^{\mathrm{a}}$ & & 1 colony & 233 & 1 colony & \\
\hline Zone 3 & $6-20$ & $1083.33 \pm 491.6^{\mathrm{a}}$ & & 2 colonies & 666 & 2 colonies & \\
\hline Zone 4 & $10-25$ & $3916.67 \pm 1068.5^{b}$ & & 0 colony & 1916 & 0 colony & \\
\hline Zone 5 & $10-23$ & $2208.33 \pm 2379.2^{\mathrm{ab}}$ & & 4 colonies & 1333 & 0 colony & \\
\hline $\mathrm{P}$-value & & 0.0123 & & & & & \\
\hline
\end{tabular}


Table 7. Legend describing level of water risk in relation to fecal coliforms.

\begin{tabular}{cccc}
\hline & No fecal coliforms $/ 100 \mathrm{ml}$ & Risk description & Color designation \\
\hline $\mathbf{1}$ & $<1$ & very low risk & \\
$\mathbf{2}$ & $10-100$ & low risk & \\
$\mathbf{3}$ & $>100$ & "high risk" or "very high risk" & \\
\hline
\end{tabular}

problematic. In this study E. coli (EPEC) was detected in zone one in a borehole water both in the dry and rainy seasons and borehole water in zone 5 were most contaminated with fecal coliforms in the dry season.

\subsection{Multivariate Analysis of Risk Factors for Boreholes and Hand Dug Wells Contamination}

Table 8. Assessment of relationship between scored hazards and pathogenic bacteria isolated from borehole water.

\begin{tabular}{|c|c|c|c|c|c|c|c|c|}
\hline & stat & $\begin{array}{l}\text { Fecal } \\
\text { coliform }\end{array}$ & E. coli 1 & $\begin{array}{c}P . \\
\text { earuginosa }\end{array}$ & $\begin{array}{c}K . \\
\text { pneumoneae }\end{array}$ & $\begin{array}{l}\text { Salmonella } \\
\quad \mathrm{sp}\end{array}$ & $\begin{array}{c}S . \\
\text { aureuse }\end{array}$ & $\begin{array}{c}S . \\
\text { pneumoneae }\end{array}$ \\
\hline \multirow{3}{*}{$\begin{array}{l}\text { Unsanitary/worn-out seal of } \\
\text { borehole pumps }\end{array}$} & odds Ratio & 1.3 & 0.4 & 0.17 & 2.5 & 0.3 & 0.67 & nvc \\
\hline & $\left(\mathrm{x}^{2}\right) \mathrm{pv}$ & $(0.8)$ & 0.8 & (0.1) 2.1 & $(0.2) 1.6$ & (0.3) 1.1 & 0.13 & $(0.04) 4.2$ \\
\hline & $95 \%(C L)$ & $0.16-9.9$ & $0.05-3.4$ & $0.13-2.1$ & $0.3-2.2$ & $0.02-3.6$ & $0.08-5.8$ & $0.92-3.3$ \\
\hline \multirow{3}{*}{$\begin{array}{l}\text { Presence of pit latrine at } 10 \mathrm{~m} \text { from } \\
\text { botre holes }\end{array}$} & odds & 2.5 & 0.25 & 1.5 & 1.3 & 3.6 & 0.4 & 2.8 \\
\hline & $\left(x^{2}\right) p v$ & (0.4) 0.7 & $(0.2) 1.6$ & (0.7) 0.1 & (0.8) 0.05 & (0.3) 1.0 & (0.5) 0.5 & (0.4) 0.6 \\
\hline & $95 \%(\mathrm{CL})$ & $0.3-21$ & $0.03-2.2$ & $0.17-13$ & $0.12-9.9$ & $0.25-46$ & $0.05-3.9$ & $0.2-40$ \\
\hline \multirow{3}{*}{$\begin{array}{l}\text { Environmental sources of pollution } \\
\text { (e.g. rubbish and surface water } \\
\text { discharge) within } 10 \mathrm{~m} \text { radius }\end{array}$} & odds Ratio & 0.5 & 0.5 & 1 & 0.16 & 0.6 & 0.3 & 1.5 \\
\hline & $\left(\mathrm{x}^{2}\right) \mathrm{pv}$ & (0.5) 0.4 & (0.5) 0.4 & (1) 00 & (0.12) 2.6 & (0.6) 0.2 & (0.2) 1.2 & (0.1) 2.5 \\
\hline & $95 \%(\mathrm{CL})$ & $0.06-4.1$ & $0.06-4.1$ & $0.12-0.9$ & $0.13-1.9$ & $0.06-5.8$ & $0.03-2.7$ & $0.95-2.3$ \\
\hline \multirow{3}{*}{ No fence around boreholes } & odds Ratio & 0.7 & 0.6 & 1 & 0.5 & 1.8 & 3.5 & 1.5 \\
\hline & $\left(\mathrm{x}^{2}\right) \mathrm{pv}$ & (0.09) 2.9 & $(0.67) 0.2$ & (1) 00 & $(0.5) 0.4$ & (0.63) 0.2 & $(0.2) 1.3$ & $(0.7) 0.9$ \\
\hline & $95 \%(C L)$ & $0.7-70.7$ & $0.073-5.4$ & $0.11-8.9$ & $0.06-4.1$ & $0.17-17.7$ & $0.37-32.9$ & $0.1-20.43$ \\
\hline \multirow{3}{*}{ Riverbed near boreholes } & odds Ratio & 2 & 0.62 & 3.5 & 0.5 & 0.4 & 3.5 & 1.4 \\
\hline & $\left(\mathrm{x}^{2}\right) \mathrm{pv}$ & $(0.5) 4$ & (0.7) 0.2 & $(0.3) 1.2$ & $(0.5) 0.4$ & $(0.5) 0.5$ & $(0.2) 1.3$ & $(0.7) 0.1$ \\
\hline & $95 \%$ & $0.2-16.6$ & $0.07-5.4$ & $0.37-32$ & $0.06-4.1$ & $0.03-5.2$ & $0.4-32$ & $0.1-20.4$ \\
\hline \multirow{3}{*}{$\begin{array}{l}\text { Lack of local management } \\
\text { committee }\end{array}$} & odds & 0.4 & 1.8 & 0.58 & 2.5 & 4.5 & 2.7 & nvc \\
\hline & $\left(\mathrm{x}^{2}\right) \mathrm{pv}$ & (0.5) 0.5 & (0.6) 0.23 & $(0.7) 0.2$ & (0.4) 0.5 & $(0.2) 1.5$ & $(0.4) 0.7$ & $(0.21) 1.4$ \\
\hline & $95 \%(C L)$ & $0.03-5.1$ & $0.2-17.7$ & $0.04-7.7$ & $0.19-32$ & $0.37-54.1$ & $0.25-28$ & $0.5-1.04$ \\
\hline \multirow{3}{*}{ Pumps manipulated with feet } & odds Ratio & 0.4 & 1.8 & 0.6 & 2.5 & 4.5 & 11 & nvc \\
\hline & $\left(\mathrm{x}^{2}\right) \mathrm{pv}$ & (0.5) 0.5 & (0.6) 0.2 & (0.7) 0.2 & (0.5) 0.5 & $(0.2) 1.5$ & $(0.01) 6.4$ & $(0.2) 1.3$ \\
\hline & $95 \%(C L)$ & $0.03-5.1$ & $0.17-17.6$ & $0.04-7.9$ & $0.19-32$ & $0.37-54$ & $1.4-174$ & $0.51-1.0$ \\
\hline \multirow{3}{*}{$\begin{array}{l}\text { Uncapped well within } 15-20 \mathrm{~m} \text { of } \\
\text { the boreholes }\end{array}$} & odds Ratio & 1.2 & 1.3 & 2.5 & 0.8 & 1.2 & 2.3 & 2 \\
\hline & $\left(\mathrm{X}^{2}\right) \mathrm{pv}$ & $(0.8) 0.04$ & (0.8) 0.05 & $(0.5) 0.5$ & $(0.8) 0.05$ & (0.9) 0.02 & $(0.5) 0.5$ & (0.6) 0.3 \\
\hline & $95 \%(C L)$ & $0.16-9.9$ & $0.13-9.9$ & $0.31-20$ & $0.1-6.3$ & $0.12-11.8$ & $0.2-20$ & $0.14-28.4$ \\
\hline
\end{tabular}


Continued

\begin{tabular}{lccccccccc}
\hline & odds Ratio & 2.5 & 0.25 & 0.4 & 0.4 & 3.6 & 0.11 & 0.5 \\
Poor drainage near the water point & $\left(\mathrm{x}^{2}\right) \mathrm{pv}$ & $(0.4) 0.7$ & $(0.2) 1.6$ & $(0.5) 0.5$ & $(0.4) 0.7$ & $(0.4) 1.0$ & $(0.6) 3.3$ & $(0.6) 2.7$ \\
& $95 \%(\mathrm{CL})$ & $0.3-29.4$ & $0.02-2.2$ & $0.05-3.9$ & $0.28-46$ & $0.008-1.4$ & $0.04-7.1$ & $0.2-9.9$ \\
& odds & 4 & 1.3 & 0.66 & 0.80 & 5.2 & 0.67 & 2 \\
& $\left(\mathrm{X}^{2}\right) \mathrm{pv}$ & $(0.2) 1.6$ & $(0.8) 0.05$ & $(0.08) 5.8$ & $(0.83) 0.05$ & $(0.19) 1.8$ & $(0.7) 0.13$ & $(0.6) 0.26$ \\
Animal and bird excreta & $95 \%(\mathrm{CL})$ & $0.44-35$ & $0.16-9.9$ & $0.08-5.8$ & $0.1-6.3$ & $0.4-68.9$ & $0.08-5.8$ & $0.14-28.4$ \\
& odds Ratio & 0.245 & 1.93 & 0.57 & 2.6 & 2.67 & 2.7 & 0.29 \\
$\begin{array}{l}\text { Other sources of contamination } \\
\text { such as wastewater dumps, within }\end{array}$ & $\left(\mathrm{x}^{2}\right) \mathrm{Pv}$ & $(0.01) 2.4$ & $(0.5) 0.4$ & $(0.6) 0.4$ & $(0.3) 1.2$ & $(0.2) 1.1$ & $(0.4) 0.6$ & $(0.3) 1.0$ \\
$\begin{array}{l}10 \mathrm{~m} \text { and frequency of cleaning the } \\
\text { environment }\end{array}$ & $95 \%(\mathrm{CL})$ & $0.04-1.5$ & $0.25-14.9$ & $0.1-3.2$ & $0.45-15.4$ & $0.43-16.4$ & $0.25-28$ & 0.025 \\
\hline
\end{tabular}

$95 \%(C L)=95 \%$ confidence level, nvc $\wedge$ Non valuable calculation.

Table 9. Assessment of relationship between scored hazards and pathogenic bacteria isolated from hand-dug well water.

\begin{tabular}{|c|c|c|c|c|c|c|c|c|c|c|}
\hline & stat & $\begin{array}{c}\text { Fecal } \\
\text { coliform }\end{array}$ & E. coli 1 & $\begin{array}{c}P . \\
\text { earuginosa }\end{array}$ & $\begin{array}{c}K . \\
\text { pneumoneae }\end{array}$ & $\begin{array}{l}\text { Salmonela } \\
\quad s p\end{array}$ & $\begin{array}{c}S . \\
\text { aureus }\end{array}$ & $\begin{array}{c}S . \\
\text { pneumoneae }\end{array}$ & $\begin{array}{l}\text { Vibrio } \\
\text { cholera }\end{array}$ & $\begin{array}{c}\text { B. } \\
\text { cepaceae }\end{array}$ \\
\hline Presence of Latrine & odds Ratio & 0.14 & 8.8 & 2 & 4 & 10 & 11 & 1.4 & 0.25 & 3.6 \\
\hline $\begin{array}{l}\text { or septic tank } \\
\text { soak-away within } 10\end{array}$ & $\left(\mathrm{x}^{2}\right) \mathrm{pv}$ & (0.04) 4.02 & $(0.05) 3.6$ & (0.4) 0.5 & (0.1) 2.3 & $(0.02) 3.4$ & $(0.03) 4$ & (0.7) 0.1 & $(0.2) 1.6$ & (0.3) 1.03 \\
\hline $\mathrm{m}$ of the wells & $95 \%(C L)$ & $0.02-1.03$ & $0.77-100$ & $0.33-11.7$ & $0.63-25$ & $1.3-78$ & $1.1-120$ & 0.16 & $0.3-2.2$ & $0.26-46.5$ \\
\hline \multirow{4}{*}{$\begin{array}{l}\text { Fencing around the } \\
\text { well inadequate to } \\
\text { keep animals away }\end{array}$} & odds Ratio & 0.5 & 1.1 & 0.6 & 2.6 & 0.4 & 2.6 & 0.5 & 10 & 3.6 \\
\hline & $\left(\mathrm{x}^{2}\right) \mathrm{pv}$ & (0.4) 0.5 & (0.9) 0.01 & (0.6) 0.2 & $(0.2) 1.1$ & (0.3) 0.9 & (0.4) 0.9 & $(0.5) 0.4$ & $(0.06) 3.6$ & (0.3) 1.01 \\
\hline & $95 \%(\mathrm{CL})$ & $0.09-3.1$ & $0.14-8.6$ & $0.11-3.7$ & $0.44-16$ & $0.06-2.7$ & $0.4-17.8$ & 0.04 & $0.78-12$ & $0.3-46.4$ \\
\hline & odds & 1.2 & 1.12 & 0.57 & 1.2 & 2.67 & 4 & 0.64 & 4 & 1.2 \\
\hline \multirow[t]{3}{*}{$\begin{array}{l}\text { Absence of apron } \\
\text { wall less than } 1 \mathrm{~m}\end{array}$} & $\left(\mathrm{x}^{2}\right) \mathrm{pv}$ & (0.8) 0.04 & (0.5) 0.4 & (0.5) 0.4 & (0.8) 0.04 & $(0.2) 1.2)$ & $(0.1) 2.6$ & (0.03) 4.5 & $(0.2) 0.6$ & (0.8) 0.02 \\
\hline & $95 \%$ & $0.22-6.7$ & $0.25-14.9$ & $0.1-3.2$ & $0.23-6.7$ & $0.43-6.4$ & $0.7-33.7$ & $0.41-0.9$ & $0.44-70.8$ & $0.4-11.9$ \\
\hline & odds Ratio & 0.8 & 2.5 & 0.9 & 1.75 & 0.7 & 3.5 & 0.37 & 0.63 & 1.8 \\
\hline \multirow[t]{3}{*}{$\begin{array}{l}\text { Poor depth and lack } \\
\text { of internal lining }\end{array}$} & $\left(\mathrm{x}^{2}\right) \mathrm{pv}$ & $(0.1)$ & (0.3) 0.8 & (0.8) 0.02 & (0.5) 0.4 & (0.6) 0.2 & $(0.2) 1.7$ & $(0.4) 0.6)$ & (0.7) 0.2 & (0.05) 3.6 \\
\hline & $95 \%(\mathrm{CL})$ & $0.14-4.5$ & $0.32-19.5$ & $0.16-5.1$ & $0.31-10$ & $0.12-11.3$ & $0.5-24.3$ & $0.03-4.4$ & $0.073-3.3$ & $1.0-3.2$ \\
\hline & odds Ratio & 0.24 & 1.9 & 0.57 & 2.62 & 2.67 & 6.39 & 0.31 & 1.6 & 2.5 \\
\hline \multirow[t]{3}{*}{$\begin{array}{l}\text { Abandoned materials } \\
\text { inside the wells }\end{array}$} & $\left(\mathrm{x}^{2}\right) \mathrm{pv}$ & (0.1) 2.3 & (0.5) 0.4 & (0.5) 0.4 & $(0.2) 1.1$ & $(0.2) 1.1$ & $(01) 6$ & (0.03) 4.4 & (0.7) 0.9 & (0.4) 0.51 \\
\hline & $95 \%(\mathrm{CL})$ & $0.39-1.5$ & $0.25-14.9$ & $0.1-3.2$ & $0.45-15.3$ & $0.43-16.3$ & $1.4-174$ & 0.41 & $0.19-13.7$ & $0.19-32.9$ \\
\hline & odds & 0.37 & 3.11 & 3.7 & 0.86 & 2.57 & 2.5 & 0.55 & 1.2 & 1.2 \\
\hline \multirow[t]{2}{*}{$\begin{array}{l}\text { Farming activities } \\
\text { near the wells }\end{array}$} & $\left(\mathrm{x}^{2}\right) \mathrm{pv}$ & $(0.2) 1.1$ & (0.3) 0.9 & (0.01) 2.03 & (0.8) 0.03 & (0.3) 0.9 & (0.8) 0.3 & (0.3) 0.5 & 0.05 & 0.024 \\
\hline & $95 \%(C L)$ & $0.6-2.3$ & $0.28-34.4$ & $0.58-23.9$ & $0.15-4.9$ & $0.37-17.8$ & $0.4-13.9$ & 0.06 & $0.16-9.9$ & $0.1-11.9$ \\
\hline \multirow{4}{*}{$\begin{array}{l}\text { Poor drainage } \\
\text { around the wells that } \\
\text { can allow water to } \\
\text { stand }\end{array}$} & odds Ratio & 0.14 & 8.8 & 4.9 & 1.75 & 3.7 & 3.5 & 0.37 & $\mathrm{ncv}$ & ncv \\
\hline & $\left(\mathrm{x}^{2}\right) \mathrm{pv}$ & (0.04) 4.1 & $(0.05) 3.6$ & $(0.01) 2.7$ & (0.5) 0.4 & (0.01) 2.03 & $(0.2) 1.6$ & (0.4) 0.6 & (0.05) 3.6 & $(0.2) 1.1$ \\
\hline & $95 \%(\mathrm{CL})$ & $0.02-1$ & $0.77-100$ & $0.70-34.3$ & $0.31-10$ & $0.59-23.9$ & $0.5-24$ & $0.03-11.3$ & $0.2-89$ & $0.42-0.9$ \\
\hline & odds Ratio & 1.9 & Nvc & 0.4 & 0.67 & 1.1 & 0.9 & 0.75 & nvc & nve \\
\hline \multirow[t]{2}{*}{ Uncapped wells } & $\left(\mathrm{x}^{2}\right) \mathrm{pv}$ & $(0.5) 0.4$ & (0.02) 2.05 & (0.3) 0.8 & (0.6) 0.2 & (0.9) 0.01 & (0.9) 0.01 & $(0.2) 1.5$ & (0.7) 3.6 & (0.16) 1.9 \\
\hline & $95 \%$ (CL) & $0.25-14.9$ & & $0.05-3.1$ & $0.08-5.1$ & $0.14-8.7$ & $0.1-7$ & 0.56 & $0.2-0.9$ & $0.41-0.9$ \\
\hline
\end{tabular}




\section{Continued}

\begin{tabular}{|c|c|c|c|c|c|c|c|c|c|c|}
\hline & odds Ratio & 0.09 & 6.67 & 1.25 & 2.63 & 2.67 & 15.75 & 0.296 & nvc & nvc \\
\hline \multirow{3}{*}{$\begin{array}{l}\text { Presence of trees } \\
\text { near the well. }\end{array}$} & $\left(\mathrm{x}^{2}\right) \mathrm{pv}$ & $(0.02) 5.9$ & (0.01) 2.8 & (0.01) 0.81 & (0.2) 1.2 & $(0.2) 1.2$ & $(0.01) 6.4$ & (0.3) 1.01 & (0.6) 3.6 & (0.2) 1.9 \\
\hline & 95\% (CL) & $0.01-0.7$ & $0.59-74.5$ & $0.22-7.08$ & $0.45-15.3$ & $0.44-16.4$ & $1.4-174$ & 0.25 & $0.51-1$ & $0.4-1$ \\
\hline & odds Ratio & 4.5 & 0.41 & 0.417 & 1.77 & 0.17 & 0.74 & 0.29 & 1 & 13 \\
\hline \multirow[t]{2}{*}{$\begin{array}{l}\text { Animal and birds } \\
\text { Faeces }\end{array}$} & $\left(x^{2}\right) p v$ & (0.1) 2.4 & 0.5 & 0.9 & 0.4 & (0.1) 2.5 & (0.8) 0.1 & (0.05) 3.8 & (1) & $(0.03) 42$ \\
\hline & $95 \%$ CL & $0.63-32.29$ & $0.04-4.6$ & $0.072-66$ & $0.21-11.12$ & $0.02-1.77$ & $0.12-4.76$ & $0.25-3.45$ & $0.11-8.9$ & $0.79-20.7$ \\
\hline $\begin{array}{l}\text { Other sources of } \\
\text { contamination }\end{array}$ & odds Ratio & 4.26 & 0.76 & 0.7 & 0.21 & 0.48 & 0.14 & 0.76 & 0.3 & 0.8 \\
\hline within $10 \mathrm{~m}$ & $\left(\mathrm{x}^{2}\right) \mathrm{Pv}$ & $(0.2) 1.5$ & $(0.2) 1.5$ & (0.7) 0.1 & $(0.3)$ & (0.5) 0.36 & (0.01) 2.7 & (1.2) 0.2 & $(0.2)$ & 1.6 \\
\hline $\begin{array}{l}\text { frequency of treating } \\
\text { and cleaning of well }\end{array}$ & $95 \%(\mathrm{CL})$ & $0.37-50.2$ & $0.52-0.96$ & $0.07-6.2$ & $0.04-5.6$ & $0.11-1.6$ & 0.58 & $0.03-22.7$ & & \\
\hline
\end{tabular}

$95 \%(C L)=95 \%$ confidence level. nvc $=$ Non Valid calculation.

Table 8 and Table 9 illustrate Multivariate analysis using odds ratio to assess the association between potential sources of contamination indicated in Table 1 and Table 2 against contamination indictors observed in water samples. These tables show odds ratios calculated for the various potential contamination factors of water along with confidence intervals and significance levels. An odd ratio of one indicates no difference between faulty and improved practices with respect to contamination; an odds ratio of less than one can also be interpreted as no difference for WHO-recommended sanitary practices, which are expected to improve water quality odds ratio above one indicates a relationship with the contamination factor in question.

In boreholes water, it was observed that Nearest latrine or a pit latrine that percolates to soil (interval $0.25-46 ; \mathrm{p}=0.3$ ); No fence around boreholes (interval $0.37-32.9, \mathrm{p}=(0.2)$ were strongly associated with contamination of bore holes with Salmonella spp and $S$. aureuse respectively but were not significant while manipulation of borehole pump with feet significantly increased the risk contamination of water with $S$. aureuse by 11 times (interval $1.4-174 ; \mathrm{p}=0.01$ ). Equally odd ratios of 4.5, 3.6, 2.3 and 1.3 in boreholes water of some factors were observed but were not statistically significant. In hand dug wells the presence of trees near the hand dug wells increased the risk of water contamination with Staphillococcus aureus by 15 times $(\mathrm{p}=0.01)$, thus indicating a very significant association with the contamination factor. Lack of fencing near hand dug wells also showed an important association with Vibrio cholera contamination (Odds $=10$, interval $0.78-12)$ but this was statistically not significant $(\mathrm{p}=0.06)$. In hand dug wells, almost all the potential contamination factors tested showed a strong relationship to the presence of contamination indicator chosen.

\section{Discussion}

\subsection{Sanitary Inspection}

Epidemiologic studies indicate that sanitary surveys have played an important 
role in determining pollution sources in water bodies. It provides the foundation required to design and implement an effective water quality sampling program and provides valuable information to assist in the interpretation of water quality data. In particular, it provides public health authorities with information to aid the selection of sampling locations, times and frequencies, in order to estimate more accurately water quality, and therefore, to allow for sound risk management decisions [12]. As part of the comprehensive and complementary risk-based assessment of drinking water quality, the purpose of the on-site visit is to identify and evaluate all existing and potential sources of microbiological contamination that could affect the safety of water in the area [13]. A higher score represents a greater risk that drinking water is contaminated by fecal pollution from the area immediately surrounding the well. The principal public health concern is the use of vulnerable groundwater aquifers and surface water without purification or disinfection measures for drinking purposes. Adamawa region is known to have $53.3 \%$ of domestic water from boreholes and $42.4 \%$ of water from hand dug wells with 364 public boreholes distributed in the seven sub-divisions of the Vina Division.

The results of risk assessment of microbial contamination in this study have shown that the locations of boreholes and hand dug wells are not adequate to prevent harvested water from contamination. And there were high counts of fecal coliform in hand dug wells water. Most of the hand dug wells sampled were within distances of $<10 \mathrm{~m}$ from potential sources of groundwater contamination. There was therefore a possibility of constant inflow of leachate of effluents from the bottom of pit latrines into the nearby wells and some boreholes water Improper sanitary practices close to water collection points resulted in a dense concentration of water sources with continuous inoculation of bacteria and other microbial pathogens into the aquifers of the area. These pathogens can spread within the groundwater system through adventive transport by flowing groundwater. Abstraction of groundwater for various uses will induce forced convection and facilitate inflow of contaminants from nearby contamination sources. A similar study was carried out by Taonameso, (2019) [14] who assessed the potential health risk to rural borehole water communities in South Africa and concluded that there was a need for environmental education.

Lack of sanitary facilities and none availability of modern latrines in most of the households in rural villages and with domestic animals kept near water collection points contributed to water contamination. Some of the community members used the bushes and streams nearby as toilets, during rainy days everything is swept into the stream causing ground water contamination. Thus, the combination of sanitary inspection and microbiological analysis of water may be useful for identifying the most important causes of control measures for well contamination, which is important to support effective and rational decision-making. This study shows that safety of boreholes and hand dug wells water in general cannot be guaranteed since it is subjected to the conditions of the infrastructure (pump and distribution system) provided. Sustainability and regular 
monitoring of boreholes and hand dug wells water is essential to prevent potential public health problems that could result in communicable disease outbreaks.

\subsection{Bacteria Load in Water Samples}

Biochemical contamination in groundwater might be enhanced by anthropogenic activities such as improper disposal of waste materials. In addition, the application of fertilizers, pesticides, and metal-containing paints play a significant contribution in the perspective of groundwater pollution. Also water sources are threatened by contamination due to increased urbanization, poor wastewater drainage and other environmental pollution caused by the lack of proper garbage collection set up. Heterotrophs are broadly defined as microorganisms that require organic carbon for growth. They include bacteria, yeasts and molds [13].

In this study, there was a variation in heterotrophic plate counts analyzed for boreholes water and hand dug wells in both seasons from all sampled locations. The total heterotrophic plate counts for all water samples were generally high exceeding the limit of $1.0 \times 10^{2} \mathrm{cfu} / \mathrm{mL}$ which is the standard for drinking water [15]. In boreholes and hand dug wells the THB counts did not fall within the standards prescribed by WHO. Similar results have been reported in total heterotrophic plate counts of boreholes water in some states of northern Nigeria [16] [17] [18]. The high values obtained could be due to poor environmental conditions and the presence of stagnant water around the boreholes, which provide an excellent breeding ground for bacteria. The presence of heterotrophic bacteria exceeding the WHO limits indicates that water samples were contaminated with bacteria that could make water unsafe for drinking. This finding agrees with similar studies by Okezi, (2019) [19] who evaluated the THB of ground water in northern states of Nigeria. The higher number of heterotrophic bacteria recorded during the rainy season could probably be due to increased surface area of the volume of water and increased carbon intake which exposes the ground water runoffs. During dry season, water contamination by heterotrophic bacteria could be caused by human activities like, washing, dipping of dirty legs or hands and cans inside the wells while fetching water or increased in population since water usually become scarce in the dry season.

Ground water which is believed to be the purest form of water because of the purification properties of the soil was found to be contaminated, due to improper construction, shallowness, animal wastes, proximity to toilet facilities, sewage, refuse dump sites, seepage or discharge from septic tanks, and various human activities [20].

\subsection{Fecal Coliform Correlation of Hand Dug Wells and Boreholes Water}

Results from groundwater samples from all wells and some boreholes indicated fecal contamination with indicator organism (fecal coliforms). Fecal contamination from cows and chickens is an issue for many water sources. Many hand dug wells are near houses which are cheaply provided to satisfy the immediate need 
of the people. Often, there are few alternative sources of potable water. Households typically keep chickens as a source of protein, and cattle are a common investment. Cattle owners need water to maintain their herd through the dry season. The emergence of communicable disease outbreaks related to water in many rural areas remains a major challenge, even though there is a vast improvement of infrastructure dedicated to accessing safe drinking water to these communities [21].

The presence of a typical Enteropathogenic E. coli (EPEC) water used in the Vina Division is a major concern because of its ability to cause acute infections. Low-economic communities are known to use water of poor quality and this is recognized internationally as an issue of critical concern to public health. The operation and management of these water supplies may be inadequate due to the limited resources and lack of awareness of factors affecting water quality [22]. Based on the WHO, (2004) [23] requirement the presence of E. coli cells in 100 $\mathrm{mL}$ of drinking water, is considered as not suitable for human consumption, unless treated. Similar studies were carried out at Dodowa in Ghana and identified high level contamination of boreholes and hand dug wells water with high levels of fecal coliforms [24]. Other studies were carried out by Mushi, (2012) [18] in Tanzania correlated risk factor contamination to $E$. coli detected in hand dug wells and boreholes water samples. Similar study indicated high level contamination of boreholes and hand dug wells water at Yola Nigeria [25]. To this effect we attributed high level of fecal coliforms counts in the dry season in our area of study to increased anthropogenic activities with relatively lower groundwater tables and conditions that enable accumulation of fecal material in water. It is reported that groundwater resources are at less risk of being contaminated by animal feces when livestock densities are low or when livestock are spread out over a wide area of land [26].

Boreholes and hand dug wells in the Vina-Division were observed to have poor drainage systems and stagnant water accumulated around the water collection points so that, underground contamination through seepage was eminent. The penetration of surface water carrying animal wastes or sewage to groundwater abstraction wells could lead to gastrointestinal illnesses as faecal material could carry various pathogenic microbes. Enterohaemorrhagic E. coli (EPEC) or Shiga toxin-producing E. coli found in humans, cattle and goats has been found in water and causing of bloody diarrhea has previously reported [27]. Each year, (EPEC) reportedly causes more than 200 million cases of diarrhoea and 380,000 deaths monthly in children in developing countries [22].

The results of this study indicate that even though boreholes are viewed as improved drinking water sources and are expected to provide safe drinking water, some of the boreholes are of very poor microbiological quality. As reported by Onda et al. (2012) [28], pathogenic bacteria and other substances from excreta in pit latrines can leach through the sub-surface soils and contaminate groundwater as seen in zone 1, both in the dry and rainy seasons.

Other observations indicated that Environmental Impact Assessment (E.I.A) 
is not done when a site for drilling boreholes was selected. This is evidenced by the number of boreholes drilled next to the potential sources of pollutants.

Several risk factors/hazards were identified to be very associated to microbial contamination of sampled sites; (Nearest latrine or a pit latrine that percolates to soil, Pumps manipulated with feet, Presence of trees near the well, Abandoned materials inside the wells). This confirms that WHO recommendations are effective as far as sanitation and water quality is concerned. It is important to note that the $\mathrm{p}$ values are large for most of these results which could indicate that these odds ratios may be a reflection of random variation in sampling. The majority of sites had contaminated water and multiple poor sanitary practices, making it difficult to isolate the effect of any individual practice. It was interesting to note that the presence of latrine close to water collection points was strongly associated with the contamination of water by fecal coliforms, E. coli 1 and some other contamination indicators chosen. This suggests that latrines could be a significant source of contamination to groundwater. The importance of surface water collecting uphill and waste within $20 \mathrm{~m}$ suggested that these represent more significant sources of microbial contamination, which would fit well with the low estimated sanitation coverage in the study areas. This rapid penetration could be as a result of the geology of the soil in the study area. This is contrary to the results obtained by Howard, 2003 [29]. In Uganda where in risk factors contributing to Microbiological Contamination of shallow groundwater did not show any strong association with fecal coliforms. These data emphasized the vulnerability of the shallow and groundwater in the Vina division to contamination and a rapidly emerging public health risk following environmental contamination of ground water. In relation to this, antibiotic resistance profile of bacteria isolated from hand dug wells and boreholes water in Vina division is very high [30] indicating a public health concern. Some of the areas in which we sampled have been known for the endemic prevalence of typhoid fever and sporadic presence of cholera epidemics. Studies have shown that Immunity resulting from repeated exposure from pathogen sources, such as drinking water, has been considered to be an important factor in the control of outbreak of many some diseases, in this area [31]. It is possible that the residents of very poor sanitary and low level of education municipalities are frequently exposed to pathogens causing typhoid fever, increasing the breeding of mosquitoes and the development of other water parasites increasing their immunity A study conducted in Conakry, Guinea, showed that there was widespread contamination of shallow dug wells and suggested that microbiological contamination was associated with poor maintenance of the wells [32].

\section{Conclusion}

Field work and laboratory experiments were conducted to identify potential sources and contamination of selected boreholes and hand-dug wells water in the Vina Division. The microbial water quality was tested through heterotrophic 
plate counts, total coliform, fecal coliforms and enthoropathogenic E. coli. There was a widespread fecal pollution of groundwater in the study area. Both hand-dug wells and boreholes are heavily loaded with fecal matter. This situation will not change unless the contaminating risk factors as we found in Table 1 and Table 2 are fixed and sanitary measures are taken. Hand-dug wells in the area seem at even greater risk to contamination compared to boreholes as indicated by multivariate analysis. Based on the WHO drinking water standards, groundwater from many sampling points was not suitable for drinking and needs to be treated before consumption. So far, a relatively limited data is available in literature for Sub Saharan Africa on potential sources of ground water contamination and sanitation and there is little or no systematic monitoring of key microbiological and chemical drinking-water-quality parameters.

\section{Limitations}

This study had difficulties in sampling repetitions, because some boreholes got damaged between sampling periods, as such could not be repeated. To solve this problem, another borehole close to the sampling area and with same geological settings was used as replacement.

\section{Recommendations}

This study shows that the microbiological contamination is high in drinking water that comes from groundwater sources in urban and rural areas. Groundwater remains an important source of water for people of this community, but measures need to be taken urgently to avoid consumption of such untreated water. It is important for the local governing body, the local councils, to come out with regulations for setting of wells and boreholes to ensure adequate distances between the sources and potential point sources of pollution and that local management committees involving women are created at each location point. A law is needed that will enforce the provision of protection zones around facilities used in abstracting groundwater for domestic use in the area. On top of that, it is important to do regular repair of broken wells and boreholes. The community needs to be educated both on activities around the drinking water sources that impair the microbial quality of groundwater and on safe handling of water obtained from these sources.

\section{Acknowledgements}

Wish to acknowledge Dr. Tanya Ngwang Vincent for his financial support.

\section{Author's Contributions}

Tangwa B.V., Ngakou A., Emmanuel N.N. conceived and designed the study.

Okah-Nnane N.H., Tangwa C.L. designed data collections and analysis tools. Tangwa B.V., Okah-Nnane N.H., Tangwa C.L. prepared the first drafted 
manuscript.

Manchang T.K., Germanus Soh B., Ngu N.V., Oumarou Palou Madi critically reviewed the manuscript.

All authors read and approved the final version of the manuscript.

\section{Funding}

No specific funding was received for this study.

\section{Conflicts of Interest}

The authors declare no conflict regarding the publication of this research.

\section{References}

[1] Lawrence, A.G., Macdonald, D.M.J., Howard, A.G., Bernett, M.H., Peddley, S., Ahmed, K.M. and Nalubeg, M.G. (2001) Guidelines for Accessing Risk to Groundwater from On-Site Sanitation. British Geological Survey. Commission Report, CR/10/142, 266.

[2] Hunter, P.R., Alan, M., MacDonald, A.M. and Carter, R.C. (2010) Water Supply and Health. PLoS Medicine, 7, e1000361. https://doi.org/10.1371/journal.pmed.1000361

[3] Galadima, A., Garba, Z.N., Leke, L., Almustapha, M.N. and Adam, I.K. (2011) Domestic Water Pollution among Local Communities in Nigeria-Causes and Consequences. European Journal of Scientific Research, 52, 592-603. https://doi.org/10.5539/jsd.v4n4p22

[4] United Nations Population Fund (2007) State of World Population-Unleashing the Potential of Urban Growth. United Nations Population Fund, New York, 104 p.

[5] Mengjo, J.W., Nche, L.A., Takeshi, O. and Kamtchueng, B.T. (2017) Effect of Diffuse Recharge and Wastewater on Groundwater Contamination in Douala, Cameroon. Environmental Earth Sciences, 76, 354.

https://doi.org/10.1007/s12665-017-6692-8

[6] Hoko, Z. (2005) An Assessment of the Water Quality of Drinking Water in Rural Districts in Zimbabwe. The Case of Gokwe South, Nkayi, Lupane, and Mwenezi Districts. Physics and Chemistry of the Earth, 30, 859-866.

http://www.researchgate.net/publication/237210161 https://doi.org/10.1016/j.pce.2005.08.031

[7] Sorensen, J.P.R., Lapworth, D.J., Nkhuwa, D.C.W., Stuart, M.E., Gooddy, D.C., Bell, R.A., Chirwa, M., Kabika, J., Liemisa, M. and Chibesa, M. (2015) Emerging Contaminants in Urban Groundwater Sources in Africa. Water Research, 72, 51-63. https://doi.org/10.1016/j.watres.2014.08.002

[8] Zeitlyn, D. (2018) A Summary of Cameroonian Administrative History. Vestiges. Traces of Record, 4, 1-13.

[9] Mamoudou, A., Nguetoum, N.C., Zolip, A. and Sevidzem, S.L. (2016) Identification and Infestation of Ticks on Cattle in the Peri-Urban Area of Ngaoundere, Cameroon. Journal of Veterinary Science and Medical Diagnosis, 4, 1-5. https://doi.org/10.4172/2325-9590.1000209

[10] Howard, G., Pedley, S., Barrett, M., Nalubega, M. and Johal, K. (2003) Risk Factors Contributing to Microbiological Contamination of Shallow Groundwater in Kampala, Uganda. Water Research, 37, 3421-3429. 
https://doi.org/10.1016/S0043-1354(03)00235-5

[11] Robins-Browne, R.M. and Hartland, E.L. (2002) Escherichia coli as a Cause of Diarrhea. Journal of Gastroenterology and Hepatology, 17, 467-475.

https://doi.org/10.1046/j.1440-1746.2002.02769.x

[12] WHO (1997) Guidelines for Drinking Water Quality, Surveillance and Control of Community Supplies. 2nd Edition, Vol. 3, World Health Organization, Geneva, 445 p.

[13] Luby, S.P., Gupta, S.K., Sheikh, M.A., Johnston, R.B., Ram, P.K. and Islam, M.S. (2008) Tubewell Water Quality and Predictors of Contamination in Three Flood-Prone Areas in Bangladesh. Journal of Applied Microbiology, 105, 1002-1008. https://doi.org/10.1111/j.1365-2672.2008.03826.x

[14] Taonameso, S., Mudau, L.S., Traoré, A.N. and Potgieter, N. (2019) Borehole Water: A Potential Health Risk to Rural Communities in South Africa. Water Supply, 19, 128-136. https://doi.org/10.2166/ws.2018.030

[15] EPA US (2009) EPA Method: 130.2: Hardness, Total (mg/L as CaCO3) (Titrimetric, EDTA). Methods for the Chemical Analysis of Water and Wastes (MCAWW) (EPA/600/4-79/020), 745 p.

[16] Erah, O.P., Akujieze, C.N. and Oteze, G.E. (2002) The Quality of Groundwater in Benin City: A Baseline Study on Inorganic Chemicals and Microbial Contaminants of Health Importance in Boreholes and Open Wells. Tropical Journal of Pharmaceutical Research, 1, 75-82. https://doi.org/10.4314/tjpr.v1i2.14587

[17] Uzoigwe, C.I. and Agwa, O.K. (2012) Microbiological Quality of Water Collected from Boreholes Sited near Refuse Dumpsites in Port Harcourt. African Journal of Biotechnology, 11, 3135-3139. https://doi.org/10.5897/AJB10.2664

[18] Adogo, L.Y., Ajiji, M.A., Anyanwu, N.C.J. and Ajide, B. (2016) Bacteriological and Physicochemical Analysis of Borehole Water in Auta Balefi Community, Nasarawa State, Nigeria. British. Microbiology Research Journal, 11, 1-7. https://doi.org/10.9734/BMRJ/2016/22360

[19] Okezie, O. (2019) Seasonal Dynamics of the Microbial and Physicochemical Characteristics of Streams and Boreholes in Uzuakoli, Eastern Nigeria. Journal of Biology and Life Science, 10, 17. https://doi.org/10.5296/jbls.v10i2.14456

[20] Mushi, T.D., Byamukama, D., Kirschner, A.K.T., Mach, R.L., Brunner, K. and Farnleitner, A.H. (2012) Sanitary Inspection of Wells Using Risk-of-Contamination Scoring Indicates a High Predictive Ability for Bacterial Faecal Pollution in the Peri-Urban Tropical Low Lands of Dar es Salaam. Journal of Water and Health, 10, 236-243. https://doi.org/10.2166/wh.2012.117

[21] UNICEF (2009) Cholera Outbreak South Africa National Outbreak Committee Situational Report (Sitrep 29) as of 12:00 hrs 11th January 2009: 1-5.

http://ochaonline.un.org/Zimbabwe

[22] WHO (2011) Guidelines for Drinking-Water Quality. World Health Organization, Geneva, 748 p.

[23] WHO (2004) Vibrio cholera. In: Guidelines for Drinking-Water Quality, 2nd Edition, Addendum: Microbiological Agents in Drinking Water, World Health Organization, Geneva, 119-142.

[24] Lutterodt, G., Foppen, J.W.A. and Uhlenbrook, S. (2014) Escherichia coli Strains Harvested from Springs in Kampala, Uganda: Cell Characterization and Transport in Saturated Porous Media. Hydrological Processes, 28, 1973-1988.

https://doi.org/10.1002/hyp.9733 
[25] Timothy, M.C., Benjamin, I.U. and David, O.P. (2007) Environmental Impact on the Quality of Water from Hand-Dug Wells in Yola Environs. Leonardo Journal of Sciences, 10, 67-76.

[26] DWAF (2004) White Paper on Basic Household and Sanitation. Pretoria, 85 p.

[27] Nguyen, R.N., Taylor, L.S., Tauschek, M. and Robins-Browne, R.M. (2006) Atypical Enteropathogenic Escherichia coli Infection and Prolonged Diarrhoea in Children. Journal of Emerging Infectious Diseases, 12, 597-603. https://doi.org/10.3201/eid1204.051112

[28] Onda, K., Jamie, B. and LoBuglio, J. (2012) Global Access to Safe Water: Accounting for Water Quality and the Resulting Impact on MDG Progress. International Journal of Environmental Research and Public Health, 9, 880-894. https://doi.org/10.3390/ijerph9030880

[29] Howard, G., et al. (2003) Risk Factors Contributing to Microbiological Contamination of Shallow Groundwater in Kampala, Uganda. Water Research, 37, 3421-3429. https://doi.org/10.1016/S0043-1354(03)00235-5

[30] Tangwa, B.V., Horline, K., Emmanuel, N.N. and Albert, N. (2019) Antimicrobial Resistance Profile of Bacteria Isolated from Boreholes and Hand Dug Wells Water in Ngaoundere Municipality of Adamawa Region in Cameroon. Advances in Microbiology, 9, 629-645. https://doi.org/10.4236/aim.2019.97039

[31] Febriani, Y., Levallois, P., Gingras, S., Gosselin, P., Majowicz, S.E. and Fleury, M.D. (2010) The Association between Farming Activities, Precipitation, and the Risk of Acute Gastrointestinal Illness in Rural Municipalities of Quebec, Canada: A Cross-Sectional Study. BMC Public Health, 10, Article No. 48.

https://doi.org/10.1186/1471-2458-10-48

[32] Gelinas, Y., Randall, H., Robidoux, L. and Schmit, J.P. (1996) Well Water Survey in Two Districts of Conakry (Republic of Guinea) and Comparison with the Piped City Water. Water Resources, 30, 2017-2026.

https://doi.org/10.1016/0043-1354(96)00040-1 